\title{
OPTIMIZATION OF A COUPLED FORCE INTENSITY BY HOMOGENIZATION METHODS
}

\author{
M. Codegone ${ }^{1}$ \\ ${ }^{1}$ Dipartimento di Matematica, Politecnico di Torino, Corso Duca degli Abruzzi 24, 10129 Torino \\ (Italy), marco.codegone@polito.it
}

\begin{abstract}
In this paper, in the framework of a problem related to an elastic non homogeneous medium, we deal with a periodic coupled force $\left(f(x) / \varepsilon^{\alpha}\right) \vec{F}(x / \varepsilon)$ with intensity of order $1 / \varepsilon^{\alpha}$. The parameter $\varepsilon$ is connected with the period of the non homogeneity of the medium and with the periodicity of the coupled force. The determination of the parameter $\alpha$ is the target of our study to obtain an effect in the microscopic equation. The homogenization technique is used in order to study the equation: $-\left(\partial / \partial x_{j}\right)\left(a_{i j k h}(x / \varepsilon) e_{k h}\left(\vec{u}^{\varepsilon, \alpha}\right)\right)=$ $\left(f(x) / \varepsilon^{\alpha}\right) F_{i}(x / \varepsilon)+G_{i}(x, x / \varepsilon)$, where $G_{i}(x, x / \varepsilon)$ is the volume applied force. The limit, when $\varepsilon \rightarrow 0$, of $\vec{u}^{\varepsilon, \alpha}(x)$, in the sense of two scale convergence, is $\left(\vec{u}^{0, \alpha}(x), \vec{u}^{1, \alpha}(x, y)\right)$ and the microscopic equation becomes: $-\left(\partial / \partial y_{j}\right)$ $\left(a_{i j k h}(y) e_{k h x}\left(\vec{u}^{0, \alpha}(x)\right)\right)-\left(\partial / \partial y_{j}\right)\left(e_{k h y}\left(\vec{u}^{1, \alpha}(x, y)\right)\right)=f(x) F_{i}(y)$ if $\alpha=1,-\left(\partial / \partial y_{j}\right)\left(a_{i j k h}(y) e_{k h x}\left(\vec{u}^{0, \alpha}(x)\right)+e_{k h y}\left(\vec{u}^{1, \alpha}(x, y)\right)\right)=0$ if $0<\alpha<1$. When $\alpha>1$ the solutions are not uniformly bounded respect to $\varepsilon$.
\end{abstract}

keywords: Coupled forces, Homogenization, Elasticity.

\section{Introduction}

In this paper, in the framework of a problem related to an elastic non homogeneous medium (see [2] and [7]), we deal with a periodic coupled force

$$
\frac{f(x)}{\varepsilon^{\alpha}} \vec{F}\left(\frac{x}{\varepsilon}\right)
$$

with intensity of order $1 / \varepsilon^{\alpha}$ (see [10], [11] and [12]). The parameter $\varepsilon$ is related to the period of the non homogeneity of the medium and to the periodicity of the coupled force. For other questions related to the homogenization theory see [3], [5] and [6]. The determination of the parameter $\alpha$ is the target of our study to obtain an effect in the microscopic equation. Let $\Omega$ be a regular domain of $\mathbf{R}^{3}$ and let $a_{i j k h}(y)$ be the symmetric, coercive and $Y$-periodic elasticity tensor:

$$
a_{i j k h}=a_{j i k h}=a_{k h i j}, \quad a_{i j k h} e_{i j} e_{k h} \geq c e_{i j} e_{i j} .
$$

Please use the following format when citing this chapter:

Codegone, M., 2006, in IFIP International Federation for Information Processing, Volume 202, Systems, Control, Modeling and Optimization, eds. Ceragioli, F., Dontchev, A., Furuta, H., Marti, K., Pandolfi, L., (Boston: Springer), pp. 115-125. 
We denote by $\vec{F}(y)$ the couple force $Y$-periodic and such that:

$$
\frac{1}{|Y|} \int_{Y} \vec{F}(y) \mathrm{d} y=0
$$

and by $f(x)$ a continuous scalar function representing the intensity of the coupled force. We indicate by $G(x, y)$ the external force $Y$-periodic in the $y$ variable. The problem is expressed by the equation:

$$
-\frac{\partial}{\partial x_{j}}\left(a_{i j k h}\left(\frac{x}{\varepsilon}\right) e_{k h}\left(\vec{u}^{\varepsilon, \alpha}\right)\right)=\frac{f(x)}{\varepsilon^{\alpha}} F_{i}\left(\frac{x}{\varepsilon}\right)+G_{i}\left(x, \frac{x}{\varepsilon}\right),
$$

in $\Omega$, with the boundary condition

$$
\left.\vec{u}^{\varepsilon, \alpha}\right|_{\partial \Omega}=0 .
$$

We prove that, if $0<\alpha \leq 1$ the solutions $u^{\varepsilon, \alpha}(x)$ are uniformly bounded:

$$
\left\|\vec{u}^{\varepsilon, \alpha}(x)\right\|_{\left(H_{0}^{1}(\Omega)\right)^{3}} \leq C .
$$

Then taking as test function:

$$
\vec{v}(x, y)=\vec{v}^{0}(x)+\varepsilon \vec{v}^{1}\left(x, \frac{x}{\varepsilon}\right)
$$

with:

$$
\vec{v}^{0}(x) \in\left(H_{0}^{1}(\Omega)\right)^{3}
$$

and with

$$
\vec{v}^{1}(x, y) \in\left(L^{2}\left(\Omega ; H_{p e r}^{1} / \mathbf{R}\right)\right)^{3}
$$

the two-convergence limit gives us the following answers: if $\alpha=1$ then

$$
\begin{gathered}
\int_{\Omega} \int_{Y} a_{i j k h}(y)\left[e_{k h x}\left(\vec{u}^{0,1}(x)\right)+e_{k h y}\left(\vec{u}^{1,1}(x, y)\right)\right] \\
{\left[e_{k h x}\left(\vec{v}^{0}(x)\right)+e_{k h y}\left(\vec{v}^{1}(x, y)\right)\right] \mathrm{d} x \mathrm{~d} y+} \\
-\int_{\Omega} \int_{Y} f(x) \vec{F}(y) \vec{v}^{1}(x, y) \mathrm{d} x \mathrm{~d} y-\int_{\Omega} \int_{Y} \vec{G}(x, y) \vec{v}^{0}(x) \mathrm{d} x \mathrm{~d} y=0,
\end{gathered}
$$

moreover if $0<\alpha<1$ then

$$
\begin{gathered}
\int_{\Omega} \int_{Y} a_{i j k h}(y)\left[e_{k h x}\left(\vec{u}^{0, \alpha}(x)\right)+e_{k h y}\left(\vec{u}^{1, \alpha}(x, y)\right)\right] \\
{\left[e_{k h x}\left(\vec{v}^{0}(x)\right)+e_{k h y}\left(\vec{v}^{1}(x, y)\right)\right] \mathrm{d} x \mathrm{~d} y+}
\end{gathered}
$$




$$
-\int_{\Omega} \int_{Y} \vec{G}(x, y) \vec{v}^{0}(x) \mathrm{d} x \mathrm{~d} y=0
$$

An integration by parts of the preceding formulae gives the following two limit problems: if $\alpha=1$ then

$$
\left\{\begin{aligned}
-\frac{\partial}{\partial y_{j}}\left[a_{i j k h}(y)\left[e_{k h x}\left(\vec{u}^{0,1}(x)\right)+e_{k h y}\left(\vec{u}^{1,1}(x, y)\right)\right]\right]-f(x) F_{i}(y)=0 \\
-\frac{\partial}{\partial x_{j}}\left[\int_{Y} a_{i j k h}(y)\left[e_{k h x}\left(\vec{u}^{0,1}(x)\right)+e_{k h y}\left(\vec{u}^{1,1}(x, y)\right)\right] \mathrm{d} y\right]+ \\
-\int_{Y} G_{i}(x, y) \mathrm{d} y=0
\end{aligned}\right.
$$

and $0<\alpha<1$ then

$$
\left\{\begin{aligned}
-\frac{\partial}{\partial y_{j}} & {\left[a_{i j k h}(y)\left[e_{k h x}\left(\vec{u}^{0, \alpha}(x)\right)+e_{k h y}\left(\vec{u}^{1, \alpha}(x, y)\right)\right]\right]=0 } \\
-\frac{\partial}{\partial x_{j}} & {\left[\int_{Y} a_{i j k h}(y)\left[e_{k h x}\left(\vec{u}^{0, \alpha}(x)\right)+e_{k h y}\left(\vec{u}^{1, \alpha}(x, y)\right)\right] \mathrm{d} y\right]+} \\
& -\int_{Y} G_{i}(x, y) \mathrm{d} y=0 .
\end{aligned}\right.
$$

In order to discuss the influence of the coupled force at the microscopic level we remarque and discuss the difference of the two microscopic equations: if $\alpha=1$

$$
-\frac{\partial}{\partial y_{j}}\left(a_{i j k h}(y) e_{k h x}\left(\vec{u}^{0,1}(x)\right)+e_{k h y}\left(\vec{u}^{1,1}(x, y)\right)\right)=f(x) F_{i}(y)
$$

and if $0<\alpha<1$

$$
-\frac{\partial}{\partial y_{j}}\left(a_{i j k h}(y) e_{k h x}\left(\vec{u}^{0, \alpha}(x)\right)+e_{k h y}\left(\vec{u}^{1, \alpha}(x, y)\right)\right)=0 .
$$

We remark that the equation (6) is a standard microscopic equation, and there the external force $f(x) F_{i}(y)$ does not appear. On the contrary the equation (5) has the external force $f(x) F_{i}(y)$, then we can conclude that the optimum coupled force that works at microscopic level is of type $\left(f(x) / \varepsilon^{\alpha}\right) \vec{F}(x / \varepsilon)$, with $\alpha=1$. In this case the macroscopic equation becomes:

$$
-\frac{\partial}{\partial x_{j}}\left(a_{i j k h}^{h o m} e_{k h x}\left(\vec{u}^{0,1}(x)\right)\right)=\Phi_{i j} \frac{\partial}{\partial x_{j}} f(x)+\tilde{G}_{i}(x)
$$

where $\Phi_{i j}$ is obtained starting from the microscopic problem and where $a_{i j k h}^{h o m}$ are the classical homogenized coefficients and

$$
\tilde{G}_{i}(x)=\frac{1}{|Y|} \int_{Y} G(x, y) \mathrm{d} x .
$$




\section{Statement of the problem with $\varepsilon>0$ and $\alpha>0$}

In order to study the equation (3), we introduce the variational formulation in the Hilbert space $\left(H_{0}^{1}(\Omega)\right)^{3}$. In this setting the existence is proved of a unique solution for $\varepsilon$ and $\alpha$ fixed and a uniform bound is obtained, with respect to $\varepsilon$ if $0<\alpha \leq 1$. In view of these targets we multiply the equation (3) by $\vec{v}^{0}(x)$ with

$$
\vec{v}^{0}(x) \in\left(H_{0}^{1}(\Omega)\right)^{3}
$$

and we integrate by parts. We get the following weak formulation:

$$
\text { find } \vec{u}^{\varepsilon, \alpha}(x) \in\left(H_{0}^{1}(\Omega)\right)^{3}
$$

such that

$$
\begin{aligned}
& \int_{\Omega} a_{i j k h}(x / \varepsilon) e_{k h x}\left(\vec{u}^{\varepsilon, \alpha}\right) e_{i j x}\left(\vec{v}^{0}\right) \mathrm{d} x= \\
& =\int_{\Omega} \frac{f(x)}{\varepsilon^{\alpha}} \vec{F}\left(\frac{x}{\varepsilon}\right) \cdot \vec{v}^{0} \mathrm{~d} x+\int_{\Omega} \vec{G}\left(x, \frac{x}{\varepsilon}\right) \cdot \vec{v}^{0} \mathrm{~d} x
\end{aligned}
$$

We suppose that $a_{i j k h}$ satisfy the hypothesis of ellipticity and symmetry:

$$
a_{i j k h}=a_{j i k h}=a_{k h i j} \quad m \xi_{i j} \xi_{i j} \leq a_{i j k h} \xi_{i j} \xi_{k h} \leq M \xi_{i j} \xi_{i j},
$$

with $m>0$. Moreover $a_{i j k h}(y)$ is $Y$-periodic of period $Y$ and

$$
f(x) \vec{F}(y), \vec{G}(x, y) \in\left(L^{2}\left(\Omega ; C_{p e r}^{0}(Y)\right)\right)^{3}
$$

where $C_{p e r}^{0}(Y)$ is the space of continuous periodic functions of period $Y$. It is known (see [1]) that, if (10) holds, then it follows that:

$$
f(x) \vec{F}(x / \varepsilon), \vec{G}(x, x / \varepsilon) \in\left(L^{2}(\Omega)\right)^{3} .
$$

By hypothesis (9), we get:

$$
m e_{i j}\left(\vec{u}^{\varepsilon, \alpha}\right) e_{i j}\left(\vec{u}^{\varepsilon, \alpha}\right) \leq \int_{\Omega} a_{i j k h}(x / \varepsilon) e_{k h}\left(\vec{u}^{\varepsilon, \alpha}\right) e_{i j}\left(\vec{u}^{\varepsilon, \alpha}\right) \mathrm{d} x,
$$

then, by Korn inequalities (see, for instance, [4] and [8]) we get:

$$
\left\|\vec{u}^{\varepsilon, \alpha}(x)\right\|_{\left(H_{0}^{1}(\Omega)\right)^{3}}^{2} \leq C \int_{\Omega} a_{i j k h}(x / \varepsilon) e_{k h}\left(\vec{u}^{\varepsilon, \alpha}\right) e_{i j}\left(\vec{u}^{\varepsilon, \alpha}\right) \mathrm{d} x .
$$

On the other hand, we need to have an estimate of the right-hand side of the equation (8):

$$
\begin{aligned}
& \left|\int_{\Omega} \frac{f(x)}{\varepsilon^{\alpha}} \vec{F}\left(\frac{x}{\varepsilon}\right) \cdot \vec{u}^{\varepsilon, \alpha} \mathrm{d} x+\int_{\Omega} \vec{G}\left(x, \frac{x}{\varepsilon}\right) \cdot \vec{u}^{\varepsilon, \alpha} \mathrm{d} x\right| \\
& \leq K\left\|\vec{u}^{\varepsilon, \alpha}\right\|_{\left(H_{0}^{1}(\Omega)\right)^{3}}
\end{aligned}
$$


Taking $\vec{G}(x, x / \varepsilon) \in\left(L^{2}\left(\Omega ; \mathcal{C}_{p e r}^{0}\right)\right)^{3}$ we get

$$
\int_{\Omega} \vec{G}\left(x, \frac{x}{\varepsilon}\right) \cdot \vec{u}^{\varepsilon, \alpha} \mathrm{d} x \leq K_{1}\left\|\vec{u}^{\varepsilon, \alpha}\right\|_{\left(H_{0}^{1}(\Omega)\right)^{3}} .
$$

The complete inequality (12) will be proved in the next section.

The estimates (11) and (12), by Lax-Milgram lemma, give the existence of a unique solution, for fixed $\varepsilon$ and $0<\alpha \leq 1$, of the weak formulation (8) and moreover give a uniform, with respect to $\varepsilon$, estimate of following norm of $\vec{u}^{\varepsilon, \alpha}$ :

$$
\left\|\vec{u}^{\varepsilon, \alpha}(x)\right\|_{\left(H_{0}^{1}(\Omega)\right)^{3}} \leq K .
$$

The estimate (13) permits us to apply the result of the two scale convergence method (see, for instance, [1], [4] and [9]) to obtain that there exist two functions

$$
\vec{u}^{0, \alpha}(x) \in\left(H_{0}^{1}(\Omega)\right)^{3} \quad \text { and } \quad \vec{u}^{1, \alpha}(x, y) \in\left(L^{2}\left(\Omega, H_{\mathrm{per}}^{1} / \mathbf{R}\right)\right)^{3}
$$

such that:

$$
\vec{u}^{\varepsilon, \alpha}(x) \rightarrow \vec{u}^{0, \alpha}(x) \quad \text { in } \quad\left(H_{0}^{1}(\Omega)\right)^{3} \quad \text { weakly }
$$

and

$$
e_{i j}\left(\vec{u}^{\varepsilon, \alpha}\right) \rightarrow e_{i j x}\left(\vec{u}^{0, \alpha}(x)\right)+e_{i j y}\left(\vec{u}^{1, \alpha}(x, y)\right)
$$

where we wrote:

$$
e_{i j x}(\vec{v})=\frac{1}{2}\left(\frac{\partial v_{i}}{\partial x_{j}}+\frac{\partial v_{j}}{\partial x_{i}}\right) \quad \text { and } \quad e_{i j y}(\vec{v})=\frac{1}{2}\left(\frac{\partial v_{i}}{\partial y_{j}}+\frac{\partial v_{j}}{\partial y_{i}}\right) .
$$

We introduce the Hilbert space

$$
\mathbf{V}=\left(H_{0}^{1}(\Omega)\right)^{3} \times\left(L^{2}\left(\Omega, H_{\mathrm{per}}^{1} / \mathbf{R}\right)\right)^{3}
$$

with the scalar product

$$
\begin{aligned}
& \left(\left(\vec{u}^{0, \alpha}, \vec{u}^{1, \alpha}\right),\left(\vec{v}^{0}, \vec{v}^{1}\right)\right)= \\
& \quad=\int_{\Omega} \int_{Y}\left[e_{i j x}\left(\vec{u}^{0, \alpha}\right)+e_{i j y}\left(\vec{u}^{1, \alpha}\right)\right]\left[e_{i j x}\left(\vec{v}^{0}\right)+e_{i j y}\left(\vec{v}^{1}\right)\right] \mathrm{d} x \mathrm{~d} y .
\end{aligned}
$$

Multiplying the equation (3) by

$$
\vec{v}(x, x / \varepsilon)=\vec{v}^{0}(x)+\varepsilon \vec{v}^{1}(x, x / \varepsilon) \quad \text { with } \quad \vec{v}(x, x / \varepsilon) \in \mathbf{V}
$$


the problem (8), in the context of the two scale convergence theory, can be written as follows

$$
\begin{gathered}
\int_{\Omega} a_{i j k h}(x / \varepsilon) e_{k h x}\left(\vec{u}^{\varepsilon, \alpha}(x)\right)\left[e_{i j x}\left(\vec{v}^{0}(x)\right)+e_{i j y}\left(\vec{v}^{1}(x, x / \varepsilon)\right)+\right. \\
\left.+\varepsilon_{i j x}\left(\vec{v}^{1}(x, x / \varepsilon)\right)\right] \mathrm{d} x= \\
=\quad \int_{\Omega} \frac{f(x)}{\varepsilon^{\alpha}} \vec{F}\left(\frac{x}{\varepsilon}\right) \cdot\left[\vec{v}^{0}+\varepsilon \vec{v}^{1}(x, x / \varepsilon)\right] \mathrm{d} x+ \\
+\int_{\Omega} \vec{G}\left(x, \frac{x}{\varepsilon}\right) \cdot\left[\vec{v}^{0}+\varepsilon \vec{v}^{1}(x, x / \varepsilon)\right] \mathrm{d} x
\end{gathered}
$$

\section{Estimate of the term with the couple force}

The coupled force $\left(f(x) / \varepsilon^{\alpha}\right) \vec{F}(x / \varepsilon)$ at the right-hand side of the equation (3) has an intensity that increases as $\varepsilon$ goes to zero. Then is not evident that the term with the coupled force may be bounded:

$$
\left|\frac{1}{\varepsilon^{\alpha}} \int_{\Omega} f(x) \vec{F}\left(\frac{x}{\varepsilon}\right) \cdot \vec{u}^{\varepsilon, \alpha} \mathrm{d} x\right| \leq C_{1}\left\|\vec{u}^{\varepsilon, \alpha}\right\|_{\left(H_{0}^{1}(\Omega)\right)^{3}}
$$

with $C_{1}$ independent of $\varepsilon$. The hypothesis (2) that $\vec{F}(x / \varepsilon)$ has zero mean value is essential. We prove the estimate (17), if

$$
0<\alpha \leq 1
$$

in the following lemma:

LEMMA 1 Let $f(x) \in \mathcal{C}^{1}(\bar{\Omega}), 0<\alpha \leq 1, \vec{F}(y) \in\left(H_{p e r}^{1}(Y)\right)^{3}$ and $\vec{u}^{\varepsilon} \in$ $\left(H_{0}^{1}(\Omega)\right)^{3}$ with $\Omega$ bounded region of $\mathbf{R}^{n}$ and $Y=[0,1]^{n}$. Moreover let $\vec{F}(y)$ be periodic with period $Y$ and suppose that the condition (2) of zero mean value holds. Then there exists a constant $C_{1}$ such that the estimate (17) is fulfilled.

Proof. We consider the vector $\vec{y}_{k} \in \mathbf{Z}^{n}$ and any translation $\vec{y}_{k}+Y$ of the fundamental period $Y=[0,1]^{n}$. We scale down the translated period with the parameter $\varepsilon$ and we say $Y_{k}^{\varepsilon}=\varepsilon\left(\vec{y}_{k}+Y\right)$. There are a finite number $N$ of the $Y_{k}^{\varepsilon}$ periods such that $\bigcup_{k=1}^{N} Y_{k}^{\varepsilon} \supseteq \Omega$. There are many period $Y_{k}^{\varepsilon}$ that are strictly included in $\Omega$, for instance $\bigcup_{k=1}^{N-T} Y_{k}^{\varepsilon} \subset \Omega$, moreover there are a number $T$ of periods $Y_{k}^{\varepsilon}$ such that $\left\{\bigcup_{k=N-T+1}^{N} Y_{k}^{\varepsilon}\right\} \cap \partial \Omega \neq \emptyset$. We now extend to zero the function $\vec{u}^{\varepsilon, \alpha}$ in the periods $Y_{k}^{\varepsilon} \cap\left\{\mathbf{R}^{n} \backslash \Omega\right\}$, with $N-T+1 \leq k \leq N$. Now 
we decompose the integral over $\Omega$ in a sum of integral over the cells $Y_{k}^{\varepsilon}$ :

$\left|\frac{1}{\varepsilon^{\alpha}} \int_{\Omega} f(x) \vec{F}\left(\frac{x}{\varepsilon}\right) \cdot \vec{u}^{\varepsilon, \alpha}(x) \mathrm{d} x\right| \leq \frac{1}{\varepsilon^{\alpha}} \sum_{k=1}^{N}\left|\int_{Y_{k}^{\varepsilon}} f(x) \vec{F}\left(\frac{x}{\varepsilon}\right) \cdot \vec{u}^{\varepsilon, \alpha}(x) \mathrm{d} x\right|$

In any period $Y_{k}^{\varepsilon}$ we choose a point $x_{k}$ where $f(x)$ attains the maximum and adding and subtracting $f\left(x_{k}\right)$ we get:

$$
\begin{aligned}
& \frac{1}{\varepsilon^{\alpha}} \sum_{k=1}^{N}\left|\int_{Y_{k}^{\varepsilon}} f(x) \vec{F}\left(\frac{x}{\varepsilon}\right) \cdot \vec{u}^{\varepsilon, \alpha}(x) \mathrm{d} x\right| \leq \\
& \leq \frac{1}{\varepsilon^{\alpha}} \sum_{k=1}^{N}\left|\int_{Y_{k}^{\varepsilon}} f\left(x_{k}\right) \vec{F}\left(\frac{x}{\varepsilon}\right) \cdot \vec{u}^{\varepsilon, \alpha}(x) \mathrm{d} x\right|+ \\
& +\frac{1}{\varepsilon^{\alpha}} \sum_{k=1}^{N} \int_{Y_{k}^{\varepsilon}}\left|f(x)-f\left(x_{k}\right)\right|\left|\vec{F}\left(\frac{x}{\varepsilon}\right)\right|\left|\cdot \vec{u}^{\varepsilon, \alpha}(x)\right| \mathrm{d} x
\end{aligned}
$$

In the $k$-cell $Y_{k}^{\varepsilon}$ we take the mean value of $\vec{u}^{\varepsilon, \alpha}(x)$ :

$$
\mathcal{M}\left(\vec{u}^{\varepsilon, \alpha}(x)\right)=\frac{1}{\left|Y_{k}^{\varepsilon}\right|} \int_{Y_{k}^{\varepsilon}} \vec{u}^{\varepsilon, \alpha}(x) \mathrm{d} x .
$$

By the condition (2) of zero mean value and by the preceding formula (20), the first term at the right-hand side of inequality (19) becomes

$$
\begin{aligned}
& \frac{1}{\varepsilon^{\alpha}} \sum_{k=1}^{N}\left|\int_{Y_{k}^{\varepsilon}} f\left(x_{k}\right) \vec{F}\left(\frac{x}{\varepsilon}\right) \cdot \vec{u}^{\varepsilon, \alpha}(x) \mathrm{d} x\right|= \\
& \quad=\frac{1}{\varepsilon^{\alpha}} \sum_{k=1}^{N}\left|\int_{Y_{k}^{\varepsilon}} f\left(x_{k}\right) \vec{F}\left(\frac{x}{\varepsilon}\right) \cdot\left(\vec{u}^{\varepsilon, \alpha}(x)-\mathcal{M}\left(\vec{u}^{\varepsilon, \alpha}(x)\right)\right) \mathrm{d} x\right|
\end{aligned}
$$

Now we apply the Cauchy-Schwartz inequality in the right-hand side of (21) to obtain

$$
\begin{aligned}
& \frac{1}{\varepsilon^{\alpha}} \sum_{k=1}^{N}\left|\int_{Y_{k}^{\varepsilon}} f\left(x_{k}\right) \vec{F}\left(\frac{x}{\varepsilon}\right) \cdot\left(\vec{u}^{\varepsilon, \alpha}(x)-\mathcal{M}\left(\vec{u}^{\varepsilon, \alpha}(x)\right)\right) \mathrm{d} x\right| \leq \\
& \quad \leq \frac{1}{\varepsilon^{\alpha}} \sum_{k=1}^{N}\left|f\left(x_{k}\right)\right|\left\|\vec{F}\left(\frac{x}{\varepsilon}\right)\right\|_{L^{2}\left(Y_{k}^{\varepsilon}\right)}\left\|\vec{u}^{\varepsilon, \alpha}(x)-\mathcal{M}\left(\vec{u}^{\varepsilon, \alpha}(x)\right)\right\|_{L^{2}\left(Y_{k}^{\varepsilon}\right)}
\end{aligned}
$$

In the last factor at the right-hand side of relation (22) we apply a Korn inequality for functions with zero mean value; it is known that in the Korn inequalities (as 
in the Poincaré-Wirtinger inequality for the scalar case, see for instance [4] and [8]) the constant $c(\varepsilon)=c \cdot \varepsilon$ is related to the measure $\varepsilon$ of the region $Y_{k}^{\varepsilon}$

$$
\begin{aligned}
& \left\|\vec{u}^{\varepsilon, \alpha}(x)-\mathcal{M}\left(\vec{u}^{\varepsilon, \alpha}(x)\right)\right\|_{L^{2}\left(Y_{k}^{\varepsilon}\right)} \leq \\
& \quad \leq c \cdot \varepsilon\left\|\sum_{i, j=1}^{n} e_{i j}\left(\vec{u}^{\varepsilon, \alpha}(x)\right)\right\|_{L^{2}\left(Y_{k}^{\varepsilon}\right)} \leq c \cdot \varepsilon\left\|\vec{u}^{\varepsilon, \alpha}(x)\right\|_{H^{1}\left(Y_{k}^{\varepsilon}\right)}
\end{aligned}
$$

The inequalities (21), (22) and (23) give us

$$
\begin{aligned}
& \frac{1}{\varepsilon^{\alpha}} \sum_{k=1}^{N}\left|\int_{Y_{k}^{\varepsilon}} f\left(x_{k}\right) \vec{F}\left(\frac{x}{\varepsilon}\right) \cdot \vec{u}^{\varepsilon, \alpha}(x) \mathrm{d} x\right| \leq \\
\leq & \frac{1}{\varepsilon^{\alpha}} \sum_{k=1}^{N}\left|f\left(x_{k}\right)\right|\left\|\vec{F}\left(\frac{x}{\varepsilon}\right)\right\|_{L^{2}\left(Y_{k}^{\varepsilon}\right)} \cdot c \cdot \varepsilon\left\|\vec{u}^{\varepsilon, \alpha}\right\|_{H^{1}\left(Y_{k}^{\varepsilon}\right)} \leq \\
\leq & \varepsilon^{1-\alpha} C_{3}\left\{\max _{x \in \Omega}|f(x)|\right\} \sum_{k=1}^{N}\left\|\vec{F}\left(\frac{x}{\varepsilon}\right)\right\|_{L^{2}\left(Y_{k}^{\varepsilon}\right)} \sum_{k=1}^{N}\left\|\vec{u}^{\varepsilon, \alpha}\right\|_{H^{1}\left(Y_{k}^{\varepsilon}\right)} \leq \\
\leq & \varepsilon^{1-\alpha} C_{4}\left\{\max _{x \in \Omega}|f(x)|\right\}\left\|\vec{F}\left(\frac{x}{\varepsilon}\right)\right\|_{L^{2}(\Omega)}\left\|\vec{u}^{\varepsilon, \alpha}\right\|_{H^{1}(\Omega)} \leq \\
\leq & \varepsilon^{1-\alpha} C_{5}\left\|\vec{u}^{\varepsilon, \alpha}(x)\right\|_{H^{1}(\Omega)}
\end{aligned}
$$

In view to obtain the estimate of the couple force, in the second term at the right-hand side of formula (19), we apply the Lipschtz inequality to the function $f(x) \in \mathcal{C}^{1}$ in the cell $Y_{k}^{\varepsilon}$

$$
\left|f(x)-f\left(x_{k}\right)\right| \leq C_{6} \cdot \varepsilon \quad x \in Y_{k}^{\varepsilon}
$$

and then we have

$$
\begin{gathered}
\frac{1}{\varepsilon^{\alpha}} \sum_{k=1}^{N} \int_{Y_{k}^{\varepsilon}}\left|f(x)-f\left(x_{k}\right)\right|\left|\vec{F}\left(\frac{x}{\varepsilon}\right)\right|\left|\vec{u}^{\varepsilon, \alpha}(x)\right| \mathrm{d} x \leq \\
\leq \frac{1}{\varepsilon^{\alpha}} \cdot C_{6} \cdot \varepsilon \sum_{k=1}^{N} \int_{Y_{k}^{\varepsilon}}\left|\vec{F}\left(\frac{x}{\varepsilon}\right)\right|\left|\vec{u}^{\varepsilon, \alpha}(x)\right| \mathrm{d} x .
\end{gathered}
$$

Adding on the cell from 1 to $N$ and applying the Cauchy-Schwartz inequality, we have:

$$
\frac{1}{\varepsilon^{\alpha}} \cdot C_{6} \cdot \varepsilon \sum_{k=1}^{N} \int_{Y_{k}^{\varepsilon}}\left|\vec{F}\left(\frac{x}{\varepsilon}\right)\right|\left|\vec{u}^{E, \alpha}(x)\right| \mathrm{d} x \leq
$$




$$
\begin{aligned}
& \leq \varepsilon^{1-\alpha} C_{6} \int_{\Omega}\left|\vec{F}\left(\frac{x}{\varepsilon}\right)\right|\left|\vec{u}^{\varepsilon, \alpha}(x)\right| \mathrm{d} x \leq \\
& \leq \varepsilon^{1-\alpha} C_{6}\left\|\vec{F}\left(\frac{x}{\varepsilon}\right)\right\|_{L^{2}(\Omega)}\left\|\vec{u}^{\varepsilon, \alpha}(x)\right\|_{L^{2}(\Omega)} \leq \\
& \leq \varepsilon^{1-\alpha} C_{6}\left\|\vec{F}\left(\frac{x}{\varepsilon}\right)\right\|_{L^{2}(\Omega)}\left\|\vec{u}^{\varepsilon, \alpha}(x)\right\|_{H^{1}(\Omega)} .
\end{aligned}
$$

The relations (24) and (26) give a uniform estimate for any $\varepsilon \in(0,1]$ if $0<$ $\alpha \leq 1$. If $\alpha>1$ the estimate is uniform only for $0<k \leq \varepsilon \leq 1$, and we can not obtain a convergent subsequence of $\vec{u}^{\varepsilon, \alpha}(x)$ as $\varepsilon$ goes to zero. The proof of the estimate of the couple force is then complete.

\section{Two-scale convergence limit}

A vector valued functions $\vec{u}^{\varepsilon, \alpha}(x)$ in $\left(L^{2}(\Omega)\right)^{3}$ is said to two-scale converge to a limit $\vec{u}^{0, \alpha}(x, y)$ belonging to $\left(L^{2}(\Omega \times Y)\right)^{3}$ if, for any function $\varphi(x, y)$ in $\left(\mathcal{D}\left(\Omega ; \mathcal{C}_{\text {per }}^{\infty}(Y)\right)\right)^{3}$, we have

$$
\lim _{\varepsilon \rightarrow 0} \int_{\Omega} \vec{u}^{\varepsilon, \alpha}(x) \varphi\left(x, \frac{x}{\varepsilon}\right) \mathrm{d} x \longrightarrow \int_{\Omega} \int_{Y} \vec{u}^{0, \alpha}(x, y) \varphi(x, y) \mathrm{d} x \mathrm{~d} y .
$$

By estimate (13), the bounded sequence $\vec{u}^{\varepsilon, \alpha}(x)$ weakly converges in $\left(H_{0}^{1}(\Omega)\right)^{3}$ to a limit $\vec{u}^{0, \alpha}(x)$. Then (see, for instance [1], [4] or [7]), if $\vec{u}^{\varepsilon, \alpha}(x)$ two-scale converges to $\vec{u}^{0, \alpha}(x)$, there exists a function $\vec{u}^{1, \alpha}(x, y)$ in $\left(L^{2}\left(\Omega, H_{\text {per }}^{1}(Y) / \mathbf{R}\right)\right)^{3}$ such that, up to a subsequence, $e_{i j x}\left(\vec{u}^{\varepsilon, \alpha}(x)\right)$ two-scale converges:

$$
e_{i j x}\left(\vec{u}^{E, \alpha}(x)\right) \rightarrow e_{i j x}\left(\vec{u}^{0, \alpha}(x)\right)+e_{i j y}\left(\vec{u}^{1, \alpha}(x, y)\right) .
$$

The weak formulation (16) of the problem (3), in the context of the two-scale convergence, gives the limit problem in the following variational form:

$$
\text { find } \quad\left(u^{0, \alpha}, u^{1, \alpha}\right) \in\left(H_{0}^{1}(\Omega)\right)^{3} \times\left(L^{2}\left(\Omega, H_{\mathrm{per}}^{1}(Y) / \mathbf{R}\right)\right)^{3}=\mathbf{V}
$$

such that

$$
\begin{gathered}
\int_{\Omega} \int_{Y} a_{i j k h}(y)\left[e_{k h x}\left(\vec{u}^{0, \alpha}(x)\right)+e_{k h y}\left(\vec{u}^{1, \alpha}(x, y)\right)\right] \\
\cdot\left[e_{k h x}\left(\vec{v}^{0}(x)\right)+e_{k h y}\left(\vec{v}^{1}(x, y)\right)\right] \mathrm{d} x \mathrm{~d} y= \\
=\delta_{\alpha 1} \int_{\Omega} \int_{Y} f(x) \vec{F}(y) \cdot \vec{v}^{1}(x, y) \mathrm{d} x \mathrm{~d} y+\int_{\Omega} \int_{Y} \vec{G}(x, y) \cdot \vec{v}^{0}(x) \mathrm{d} x \mathrm{~d} y
\end{gathered}
$$


$\forall\left(v_{0}, v_{1}\right) \in \mathbf{V}$ and where

$$
\delta_{\alpha 1}=\left\{\begin{array}{lll}
1 & \text { if } & \alpha=1 \\
0 & \text { if } & \alpha \neq 1
\end{array}\right.
$$

By the coercivity (9) of the bilinear form defined by the left-hand side of (27), we can apply the Lax-Milgram lemma and we have a well posed formulation of the limit two-scale problem. Integrating by parts the equation (27) in $x$ and $y$ and choosing $\vec{v}^{0}(x)=0$ we get the microscopic equation

$$
-\frac{\partial}{\partial y_{j}}\left\{a_{i j k h}(y)\left[e_{k h x}\left(\vec{u}^{0, \alpha}(x)\right)+e_{k h y}\left(\vec{u}^{1, \alpha}(x, y)\right)\right]\right\}=f(x) \vec{F}_{i}(y)
$$

By the linearity of the equation (28), we solve the following problem in the period $Y$

$$
\begin{cases}-\frac{\partial}{\partial y_{j}}\left[a_{i j k h}(y) e_{k h y}\left(\vec{w}^{l m, \alpha}(y)\right)\right]=\frac{\partial a_{i j l m}(y)}{\partial y_{j}} & \text { in } Y \\ \vec{w}^{l m, \alpha}(y) & Y \text {-periodic }\end{cases}
$$

and, if $\alpha=1$, we solve the further equation:

$$
\begin{cases}-\frac{\partial}{\partial y_{j}}\left[a_{i j k h}(y) e_{k h y}\left(\vec{v}^{\alpha}(y)\right)\right]=\vec{F}_{i}(y) & \text { in } Y \\ \vec{v}^{\alpha}(y) & Y \text {-periodic }\end{cases}
$$

The solution of problems (29) and (30) gives the solution of the microscopic equation (28)

$$
\vec{u}^{1, \alpha}(x, y)=e_{k h x}\left(\vec{u}^{0, \alpha}(x)\right) \vec{w}^{k h, \alpha}(y)+\delta_{\alpha 1} f(x) \vec{v}^{\alpha}(y)
$$

We remark that, at the microscopic level, the couple force has, if $\alpha=1$, an important role.

As in classical homogenization we define

$$
a_{i j k h}^{\mathrm{hom}, \alpha}=\int_{Y} a_{i j l m}(y)\left[\delta_{l k} \delta_{m h}+e_{l m y}\left(\vec{w}^{k h, \alpha}(y)\right)\right] \mathrm{d} y
$$

Moreover we define

$$
\Phi_{i j}^{\alpha}=\int_{Y} a_{i j k h}(y) e_{k h y}\left(\vec{v}^{\alpha}\right) \mathrm{d} y
$$


At this point we write the limit homogenized equation

$$
\begin{cases}-\frac{\partial}{\partial x_{j}}\left[a_{i j k h}^{\text {hom, } \alpha}(y) e_{k h x}\left(\vec{u}^{0, \alpha}(x)\right)\right]= & \\ =\delta_{\alpha 1} \Phi_{i j}^{\alpha} \frac{\partial}{\partial x_{j}}(f(x))+\int_{Y} \vec{G}_{i}(x, y) & \text { in } \Omega \\ \vec{u}^{0, \alpha}(x)=0 & \text { on } \partial \Omega\end{cases}
$$

REMARK 2 We remark that if $a_{i j k h}(y)=\bar{a}_{i j k h}$ is constant in the period $Y$, then $\Phi_{i j}^{\alpha}$ in formula (33) is zero. In same way if the intensity $f(x)$ of the couple force is constant, then $\Phi_{i j}^{\alpha}$ in formula (33) is zero. Moreover if $0<\alpha<1$, then $\Phi_{i j}^{\alpha}$ in formula (33) is zero. Then the effect of the force $\left(f(x) / \varepsilon^{\alpha}\right) F(y)$ may work at the microscopic level, if the medium is non homogeneous and $f(x)$ is non constant and if $\alpha=1$.

\section{References}

[1] G. Allaire. Homogenization and two scale convergence. SIAM J. Math. Anal. 23:1482$1518,1992$.

[2] A.Bensoussan, J.L. Lions, G. Papanicolaou. Asymptotic Analysis for Periodic Structures. North-Holland, 1978.

[3] V. Chiadò Piat, M. Codegone. Scattering problem in a perforated domain. Rev. R. Acad. Cien. Serie A Mat. 97:447-454, 2003.

[4] D. Cioranescu, P. Donato. An Introduction to Homogenization. Oxford University Press, Oxford, 1999.

[5] M. Codegone. Problème d'homogénéisation en théorie de la diffraction. C.R. Acad.Sc. Paris. 288:387-389, 1979.

[6] M. Codegone. G-Convergence and Scattering Problems. Boll. U.M.I. 6.1-A:367-375, 1982.

[7] V.V. Jikov, S.M. Kozlov, O.A. Oleinik, Homogenization of Differential Operators and Integral Functionals. Springer-Verlag, Berlin Heidelberg, 1994.

[8] O.A. Oleinik, A.S. Shamaev, G.A. Yosifian. Mathematical problems in elasticity and homogenization. Studies in mathematics and its applications, Elsevier Science Publishers, Amsterdam, 1992.

[9] G. Nguetseng. A general convergence result for a functional related to the theory of homogenization. SIAM J. Math. Anal. 20:608-623, 1989.

[10] J. Sanchez-Hubert, E. Sanchez-Palencia. Vibration and Coupling of Continuous Systems Asymptotic Methods. Springer-Verlag, Berlin, 1989.

[11] E. Sanchez-Palencia. Comportement local et macroscopique d'un type de milieux physiques hétérogènes. Internat. J. Engrg. Sci. 12:331-351, 1974.

[12] E. Sanchez-Palencia. Non Homogeneous Media and Vibration Theory. Lecture Notes in Physics, Springer-Verlag, Berlin, 1980. 